Afrika Statistika

Afrika Statistika

Vol. 9, 2014, pages 647-658.

DOI: http://dx.doi.org/1016929.as.2014.647.59

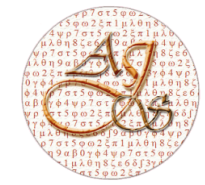

ISSN 2316-090X

\title{
On the stability of estimation of $\operatorname{AR}(1)$ coefficient in the presence of contaminated exponential innovations
}

\author{
Hocine Fellag*, Lynda Atil and Cherifa Belkacem \\ Laboratory of Pure and Applied Mathematics, Faculty of Sciences. University of Tizi Ouzou, Tizi- \\ Ouzou. 15000. Algeria
}

Received 25 May 1014; Accepted 25 September 1014

Copyright (c) 2014, Afrika Statistika. All rights reserved

\begin{abstract}
The aim of our paper is to present an exhaustive study of the estimation of first order autoregressive models with exponential white noise under innovation contamination. Some theoretical aspects and Monte Carlo results are presented in the study of the stability of this estimator when the model is contaminated. Using the methodology of Andẽl (1988) based on the mean stationarity of the process, we prove that the maximum likelihood estimator of the parameter is asymptotically stable with respect to the bias and the mean square error. Also, some results of the small sample case are obtained.

Résumé. Le but de ce travail porte sur l'estimation d'un modèle autorègressif ayant un bruit exponentiel contaminé. En utilisant la mthode d'approximation d'Andẽl (1988) base sur la stationnarit en moyenne du processus, nous prouvons, moyennant des rsultats analytiques et numriques, que le biais et l'cart quadratique moyen de l'estimateur du maximum de vraisemblance du paramtre sont asymptotiquement stables.
\end{abstract}

Key words: Autoregressive; Contamination; Exponential; Estimation; Stability.

AMS 2000 Mathematics Subject Classification : Primary 62F11; Secondary 62M10.

\section{The model}

Consider the first order autoregressive process of the form

$$
X_{t}=\rho X_{t-1}+\varepsilon_{t}, \quad t=0,1, \ldots, n .
$$

\footnotetext{
${ }^{*}$ Corresponding author Hocine Fellag: hfellag@yahoo.com Lynda Atil : atillynda@yahoo.fr

Cherifa Belkacem: cherifabelkacem@yahoo.fr
} 
H. Fellag, L. Atil and C. Belkacem, Afrika Statistika, Vol. 9, 2014, pages 647-658. On the stability of estimation of $\mathrm{AR}(1)$ coefficient in the presence of contaminated exponential innovations.

648

Where the $\varepsilon_{t}$ 's are independently distributed according to an exponential distribution $\operatorname{Exp}(1)$, i.e., the density of $\varepsilon_{t}$ is $f_{\varepsilon_{t}}(y)=e^{-y}$ with $y>0$. This model is useful for modeling a wide range of phenomena which do not allow negative values such as water quality analysis and hydrology (see, for example, Gaver and Lewis, 1980). Many authors have studied this model. Bell and Smith (1986) studied the estimating and testing problem on $\rho$. They proved almost sure consistency for the maximum likelihood estimator (MLE) of $\rho$ whereas Davis and McCormick (1989) found the limiting distribution. Turkman (1990) proposed a bayesian estimator of $\rho$ for the same model. This work was generalized by Fellag and Ibazizen (2003). Also, Fellag and Ibazizen (2001) presented some results concerning the bias of the MLE when the innovations are distributed according to the exponential power distribution.

Nielsen and Shephard (2003) derived the exact distribution of the maximum likelihood estimator of a first-order linear autoregression with an exponential disturbance term. Also, they proved that this estimator is consistent. However, the authors affirmed that, in practice, this type of model has some significant disadvantages for the estimator of $\rho$, may be very sensitive to small amounts of mismeasurement.

Now, assume that $0 \leq \rho \leq 1$ and all what we observe is a segment of the process

$$
X_{1}, X_{2}, \ldots, X_{n}, \quad n \text { fixed }
$$

with $\rho$ unknown and is to be estimated.

Andẽl (1988), in order to obtain the mean stationarity of the process, we assume that $X_{0}$ is distributed according to $\operatorname{Exp}(1-\rho)$. The maximum likelihood estimator for $\rho$ is

$$
\widehat{\rho}=\min _{2 \leq t \leq n}\left(X_{t} / X_{t-1}\right)=\rho+\min _{2 \leq t \leq n} \frac{\varepsilon_{t}}{X_{t-1}} .
$$

In Andẽl and Zvãra (1988), tables of the bias and variance of $\hat{\rho}$ are given using numerical approximations. Moreover, Andẽl (1988) considered the following modification. Since the process is stationary with mean $m=1 /(1-\rho)$, he proposed to substitute $m$ for $X_{t-1}$ in (3). Then the estimator becomes

$$
\widehat{\rho}^{*}=\rho+\frac{1}{m} \min _{2 \leq t \leq n} \varepsilon_{t} .
$$

Andẽl (1988) found then the distribution for this new modified estimator of $\rho$. Consistency results for higher-order processes are given by Andẽl (1988) and An and Huang (1993).

The problem of contamination in this kind of models can be considered in many ways. Fellag (2001) studied the behavior of the MLE under innovation outlier contamination.

In this paper, we propose a theoretical approximation of the bias and the mean square error (MSE) according to Andẽl's methodology . Also, we prove, using Monte carlo study that this approximation is satisfactory. Then, this allows us to present some results concerning the robustness of the bias and the MSE of $\widehat{\rho}^{*}$ when the innovations are contaminated according to the model of Kale and Sinha (1969). Notice that, when a contaminant occur in the distribution of the white noise, all the future of the process is deviated. Then, if the estimator is influenced by this outlier, the estimation of the parameter can become useless.

Journal home page: www.jafristat.net 
H. Fellag, L. Atil and C. Belkacem, Afrika Statistika, Vol. 9, 2014, pages 647-658. On the stability of estimation of $\mathrm{AR}(1)$ coefficient in the presence of contaminated exponential innovations.

649

This paper is outlined as follows: the second section presents the method of Andẽl applied to the exponential model. In the third section, the approximations of the bias and the MSE in the contaminated model are presented. In this section one can remark the validity of this approximation. An exhaustive numerical study is presented in the fourth section.

\section{Exponential case}

Let $B(\rho)$ and $M S E(\rho)$ be the bias and the mean squared error of the modified estimator of Andẽl $\widehat{\rho}^{*}$ in model (1) respectively.

So, we have

$$
B(\rho)=E\left(\widehat{\rho}^{*}-\rho\right)=E\left(\widehat{\rho}^{*}\right)-\rho
$$

and,

$$
M S E(\rho)=B(\rho)^{2}+\operatorname{Var}\left(\widehat{\rho}^{*}-\rho\right) .
$$

Therefore, in order to obtain the formulas of the bias and the MSE in the exponential case, we need to know the distribution of $\widehat{\rho}^{*}-\rho$. We proceed as follows,

$$
\begin{gathered}
p\left[\left(\widehat{\rho}^{*}-\rho\right)<y\right]=1-p\left[\left(\widehat{\rho}^{*}-\rho\right)>y\right]=1-p\left[\left(\min _{2 \leq t \leq n} \frac{\varepsilon_{t}}{m}\right)>y\right], \\
p\left[\left(\widehat{\rho}^{*}-\rho\right)<y\right]=1-p\left[\left(\min _{2 \leq t \leq n} \varepsilon_{t}\right)>y m\right]=1-\exp \{-y m(n-1)\} .
\end{gathered}
$$

Consequently, let $G$ be the distribution of $Y=\left(\widehat{\rho}^{*}-\rho\right)$ which will be given by the following formula,

$$
G(y)= \begin{cases}1-\exp \{-y m(n-1)\} & y>0 \\ 0 & \text { else }\end{cases}
$$

So $\left(\widehat{\rho}^{*}-\rho\right)$ follows an exponential distribution with parameter $\lambda=m(n-1)$, then the value of the bias is

$$
B(\rho)=\frac{1-\rho}{n-1}
$$

Since the variance of $\left(\widehat{\rho}^{*}-\rho\right)$ is equal to $\left(\frac{1-\rho}{n-1}\right)^{2}$, we can obtain, from the formula (6), the value of the MSE given by

$$
M S E(\rho)=2\left(\frac{1-\rho}{n-1}\right)^{2} \text {. }
$$

\section{Contaminated exponential case}

Consider the contaminated model (Kale and Sinha (1969))

$$
X_{t}=\rho X_{t-1}+\varepsilon_{t}, \quad t=0,1, \ldots, n .
$$

such that

$$
\left\{\begin{array}{l}
\varepsilon_{t} \sim \operatorname{Exp}(1), \quad \forall t \neq k, \quad t=0,1, \ldots, n . \\
\varepsilon_{t} \sim \operatorname{Exp}\left(\frac{1}{\alpha}\right), \text { if } t=k
\end{array}\right.
$$

Journal home page: www.jafristat.net 
H. Fellag, L. Atil and C. Belkacem, Afrika Statistika, Vol. 9, 2014, pages 647-658. On the stability of estimation of $\mathrm{AR}(1)$ coefficient in the presence of contaminated exponential innovations.

where $0<\alpha \leq 1$. Let be the $\widetilde{\rho}$ the modified estimator of $\widehat{\rho}^{*}$ obtained in the model (10). It means that

$$
\widetilde{\rho}-\rho=\min \left(\frac{\varepsilon_{2}}{E\left(X_{1}\right)}, \frac{\varepsilon_{3}}{E\left(X_{2}\right)}, \ldots, \frac{\varepsilon_{k-1}}{E\left(X_{k-2}\right)}, \frac{\varepsilon_{k}}{E\left(X_{k-1}\right)}, \frac{\varepsilon_{k+1}}{E\left(X_{k}\right)}, \ldots, \frac{\varepsilon_{n}}{E\left(X_{n-1}\right)}\right) .
$$

Then, the bias and the MSE of $\widetilde{\rho}$ will be given by the following proposition.

Proposition 1. In the model (10), the exact bias of $\tilde{\rho}$ is equal to

$$
\widetilde{B}(\rho)=\frac{\alpha}{\alpha^{2}\left(1+\rho m\left(1-\rho^{n-k-1}\right)\right)+\alpha m\left[(n-3+\rho)-\rho\left(1-\rho^{n-k-1}\right)\right]+m}
$$

and the mean squared error is

$$
\widetilde{M S E}(\rho)=2\left[\frac{\alpha}{\alpha^{2}\left(1+\rho m\left(1-\rho^{n-k-1}\right)\right)+\alpha m\left[(n-3+\rho)-\rho\left(1-\rho^{n-k-1}\right)\right]+m}\right]^{2}
$$

such that,

$$
m=\frac{1}{1-\rho} .
$$

Proof. First, we need the distribution of $(\widetilde{\rho}-\rho)$,

$$
\begin{aligned}
P[ & (\tilde{\rho}-\rho)<x]=1-P[(\widetilde{\rho}-\rho)>x] \\
= & {\left[\min \left(\frac{\varepsilon_{2}}{E\left(X_{1}\right)}, \frac{\varepsilon_{3}}{E\left(X_{2}\right)}, \ldots, \frac{\varepsilon_{k-1}}{E\left(X_{k-2}\right)}, \frac{\varepsilon_{k}}{E\left(X_{k-1}\right)}, \frac{\varepsilon_{k+1}}{E\left(X_{k}\right)}, \ldots, \frac{\varepsilon_{n}}{E\left(X_{n-1}\right)}\right)>x\right] } \\
= & 1-\exp \left\{-x\left(E\left(X_{1}\right)+E\left(X_{2}\right)+\ldots+E\left(X_{k-2}\right)+\frac{E\left(X_{k-1}\right)}{\alpha}+E\left(X_{k}\right)+\ldots+E\left(X_{n-1}\right)\right)\right\} .
\end{aligned}
$$

Therefore, the distribution of $(\widetilde{\rho}-\rho)$ is

$$
(\widetilde{\rho}-\rho) \sim \exp \left[m(k-2)+m / \alpha+\rho m+\alpha+\sum_{t=k+1}^{n-1} \theta_{t}\right]
$$

with

$$
\theta_{t}=\rho^{t-k+1} m+\sum_{i=0}^{t-k-1} \rho^{i}+\rho^{t-k} \alpha
$$

And consequently the bias of the contaminated estimator is given by

$$
\widetilde{B(\rho)}=\frac{\alpha}{\alpha^{2}\left(1+\rho m\left(1-\rho^{n-k-1}\right)\right)+\alpha m\left[(n-3+\rho)-\rho\left(1-\rho^{n-k-1}\right)\right]+m}
$$

and the mean squared error is

$$
\widetilde{M S E}(\rho)=2\left[\frac{\alpha}{\alpha^{2}\left(1+\rho m\left(1-\rho^{n-k-1}\right)\right)+\alpha m\left[(n-3+\rho)-\rho\left(1-\rho^{n-k-1}\right)\right]+m}\right]^{2}
$$

This completes the proof.

Journal home page: www.jafristat.net 
H. Fellag, L. Atil and C. Belkacem, Afrika Statistika, Vol. 9, 2014, pages 647-658. On the stability of estimation of $\mathrm{AR}(1)$ coefficient in the presence of contaminated exponential innovations.

651

The Table 1 compares the theoretical approximation of the bias (formula (11)) and the simulated bias of the ML estimator $\hat{\rho}$, as well as the values of the theoretical MSE (formula (12)) and of the simulated MSE of the ML estimator $\hat{\rho}$. Then, some values are proposed for $n=20,100,500, \alpha=0.1,0.5,0.9,1.0$ and $\rho=0.2,0.6,0.8$. We assume that the contaminant occurs at a specified time, say $k=[n / 2]$ where $[m]$ means the integer part of the number $m$. One can note that $\alpha=1$ represents the noncontaminated case.

Surprisingly, we notice that the values given by the formulas (11) and (12) are very similar to the simulated values of maximum likelihood estimator $\hat{\rho}$ based on 1.000.000 repetitions. Then, one can say that the formulas (11) and (12) can be considered as good approximations of the bias and the MSE of the MLE. That's why, throughout the remainder of this paper, we will use these formulas only.

Moreover, in all our simulations and computations, we noticed that the position of the contaminant has no effect on the values of the bias and MSE as given in the following Table 2 obtained with $n=100$.

In order to illustrate graphically the behavior of the bias and the MSE of the modified estimator of $\rho$ when $\alpha$ varies, the following figures are given. The figures 1 and 2 present the variation of the approximate bias and the approximate MSE for $n=20,50,250$ respectively.
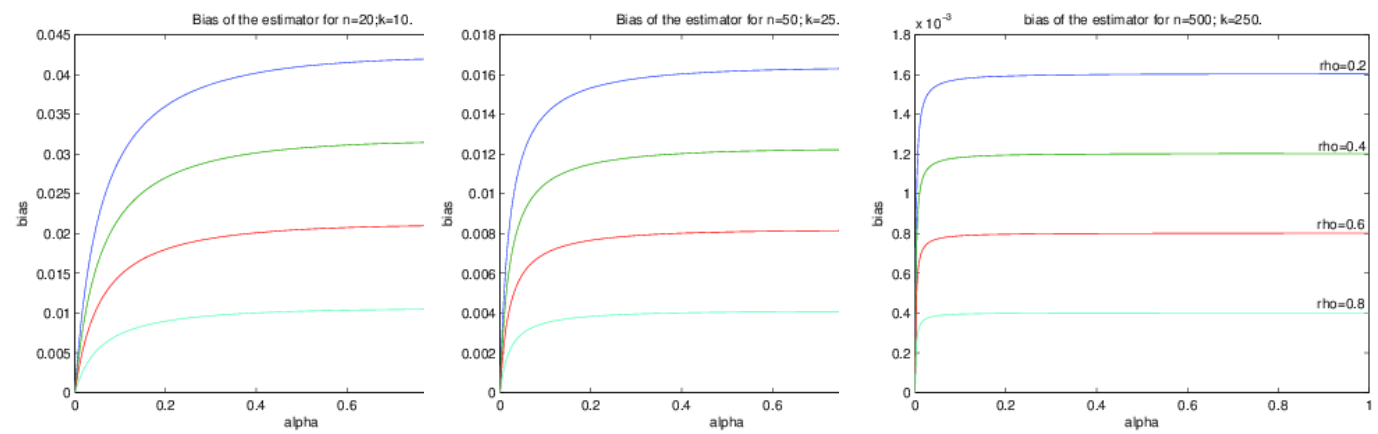

Fig. 1. Variation of the approximate bias with $\alpha$

\section{Numerical study}

Now, we propose to study the stability of the estimator of $\rho$ using the measure of robustness introduced by Zieliñski (1977) as follows: We consider the statistical model with exponential white noise denoted by

$$
M_{\alpha}=\left(\boldsymbol{R}^{+} ; \mathcal{B}^{+} ; P_{\alpha}\right)
$$


H. Fellag, L. Atil and C. Belkacem, Afrika Statistika, Vol. 9, 2014, pages 647-658. On the stability of estimation of $\operatorname{AR}(1)$ coefficient in the presence of contaminated exponential innovations. 652

\begin{tabular}{|c|c|c|c|c|c|c|}
\hline $\mathrm{n}$ & $\rho$ & $\alpha$ & Approximate bias & simulated bias & Approximate MSE & simulated MSE \\
\hline \multirow{12}{*}{20} & \multirow{4}{*}{0.2} & 0.1 & 0.0295 & 0.0306 & 0.0017 & 0.0019 \\
\hline & & 0.5 & 0.0410 & 0.0394 & 0.0034 & 0.0028 \\
\hline & & 0.9 & 0.0421 & 0.0390 & 0.0035 & 0.0030 \\
\hline & & 1.0 & 0.0421 & 0.0404 & 0.0035 & 0.0031 \\
\hline & \multirow{4}{*}{0.6} & 0.1 & 0.0148 & 0.0153 & $0.4355^{*} 10^{-3}$ & $0.4908^{*} 10^{-3}$ \\
\hline & & 0.5 & 0.0205 & 0.0202 & $0.8413^{*} 10^{-3}$ & $0.7507^{*} 10^{-3}$ \\
\hline & & 0.9 & 0.0210 & 0.0197 & $0.8853^{*} 10^{-3}$ & $0.7671^{*} 10^{-3}$ \\
\hline & & 1.0 & 0.0211 & 0.0199 & $0.8864 * 10^{-3}$ & $0.7647^{*} 10^{-3}$ \\
\hline & \multirow{4}{*}{0.8} & 0.1 & 0.0074 & 0.0081 & $0.1082^{*} 10^{-3}$ & $0.1454^{*} 10^{-3}$ \\
\hline & & 0.5 & 0.0102 & 0.0105 & $0.2092^{*} 10^{-3}$ & $0.2093 * 10^{-3}$ \\
\hline & & 0.9 & 0.0105 & 0.0102 & $0.2211^{*} 10^{-3}$ & $0.2151^{*} 10^{-3}$ \\
\hline & & 1.0 & 0.0105 & 0.0103 & $0.2216^{*} 10^{-3}$ & $0.2167^{*} 10^{-3}$ \\
\hline \multirow{12}{*}{100} & \multirow{4}{*}{0.2} & 0.1 & 0.0075 & 0.0077 & $0.1116^{*} 10^{-3}$ & $0.1150 * 10^{-3}$ \\
\hline & & 0.5 & 0.0080 & 0.0076 & $0.1293^{*} 10^{-3}$ & $0.1153^{*} 10^{-3}$ \\
\hline & & 0.9 & 0.0081 & 0.0082 & $0.1306^{*} 10^{-3}$ & $0.1388^{*} 10^{-3}$ \\
\hline & & 1.0 & 0.0081 & 0.0081 & $0.1306^{*} 10^{-3}$ & $0.1314^{*} 10^{-3}$ \\
\hline & \multirow{4}{*}{0.6} & 0.1 & 0.0037 & 0.0039 & $0.2790^{*} 10^{-4}$ & $0.3001^{*} 10^{-4}$ \\
\hline & & 0.5 & 0.0040 & 0.0038 & $0.3232^{*} 10^{-4}$ & $0.2817^{*} 10^{-4}$ \\
\hline & & 0.9 & 0.0040 & 0.0040 & $0.3264^{*} 10^{-4}$ & $0.3306^{*} 10^{-4}$ \\
\hline & & 1.0 & 0.0040 & 0.0040 & $0.3265^{*} 10^{-4}$ & $0.3152^{*} 10^{-4}$ \\
\hline & \multirow{4}{*}{0.8} & 0.1 & 0.0019 & 0.0019 & $0.6974 * 10^{-5}$ & $0.7408^{*} 10^{-5}$ \\
\hline & & 0.5 & 0.0020 & 0.0019 & $0.8081^{*} 10^{-5}$ & $0.7396 * 10^{-5}$ \\
\hline & & 0.9 & 0.0020 & 0.0020 & $0.8161^{*} 10^{-5}$ & $0.8032^{*} 10^{-5}$ \\
\hline & & 1.0 & 0.0020 & 0.0020 & $0.8162 * 10^{-5}$ & $0.7826^{*} 10^{-5}$ \\
\hline \multirow{12}{*}{500} & \multirow{4}{*}{0.2} & 0.1 & 0.0016 & 0.0015 & $0.4978^{*} 10^{-5}$ & $0.4632 * 10^{-5}$ \\
\hline & & 0.5 & 0.0016 & 0.0016 & $0.5130 * 10^{-5}$ & $0.4999 * 10^{-5}$ \\
\hline & & 0.9 & 0.0016 & 0.0015 & $0.5140^{*} 10^{-5}$ & $0.4645^{*} 10^{-5}$ \\
\hline & & 1.0 & 0.0016 & 0.0015 & $0.5141^{*} 10^{-5}$ & $0.4935^{*} 10^{-5}$ \\
\hline & \multirow{4}{*}{0.6} & 0.1 & $0.7888^{*} 10^{-3}$ & $0.7704^{*} 10^{-3}$ & $0.1244^{*} 10^{-5}$ & $0.1153^{*} 10^{-5}$ \\
\hline & & 0.5 & $0.8008^{*} 10^{-3}$ & $0.7917^{*} 10^{-3}$ & $0.1283^{*} 10^{-5}$ & $0.1263^{*} 10^{-5}$ \\
\hline & & 0.9 & $0.8016^{*} 10^{-3}$ & $0.7635^{*} 10^{-3}$ & $0.1285^{*} 10^{-5}$ & $0.1149 * 10^{-5}$ \\
\hline & & 1.0 & $0.8016^{*} 10^{-3}$ & $0.7665^{*} 10^{-3}$ & $0.1285^{*} 10^{-5}$ & $0.1203^{*} 10^{-5}$ \\
\hline & \multirow{4}{*}{0.8} & 0.1 & $0.3944^{*} 10^{-3}$ & $0.3828^{*} 10^{-3}$ & $0.3111^{*} 10^{-6}$ & $0.2810^{*} 10^{-6}$ \\
\hline & & 0.5 & $0.4004^{*} 10^{-3}$ & $0.4000 * 10^{-3}$ & $0.3206^{*} 10^{-6}$ & $0.3273^{*} 10^{-6}$ \\
\hline & & 0.9 & $0.4008^{*} 10^{-3}$ & $0.3850 * 10^{-3}$ & $0.3212^{*} 10^{-6}$ & $0.3005^{*} 10^{-6}$ \\
\hline & & 1.0 & $0.4008^{*} 10^{-3}$ & $0.3894^{*} 10^{-3}$ & $0.3213^{*} 10^{-6}$ & $0.3259 * 10^{-6}$ \\
\hline
\end{tabular}

Table 1. Approximate and simulated estimated values of the bias and MSE for $\rho=$ $0.2,0.6,0.8$

where $\mathcal{P}_{\alpha}$ means that in the model (1), the $k^{\text {th }}$ innovation is distributed according to $\operatorname{Exp}(1 / \alpha) \cdot M_{\alpha}$ is called the supermodel. One can note that $M_{1}$ is obtained when the errors are noncontaminated. The measure of robustness is established with respect to the bias and the MSE. The first measure is defined as follows :

$$
\operatorname{rbias}_{\left[\alpha_{1}, \alpha_{2}\right]}(\rho)=\sup _{\alpha_{1} \leq \alpha \leq \alpha_{2}} \widetilde{B}(\rho, \alpha)-\inf _{\alpha_{1} \leq \alpha \leq \alpha_{2}} \widetilde{B}(\rho, \alpha)
$$


H. Fellag, L. Atil and C. Belkacem, Afrika Statistika, Vol. 9, 2014, pages 647-658. On the stability of estimation of $\operatorname{AR}(1)$ coefficient in the presence of contaminated exponential innovations. 653

\begin{tabular}{|c|c||c|c||c|c||c|c|}
\hline \multicolumn{2}{|c||}{} & \multicolumn{2}{c||}{$\alpha=0.2$} & \multicolumn{2}{c||}{$\alpha=0.5$} & \multicolumn{2}{c|}{$\alpha=0.8$} \\
\hline$\rho$ & $\mathrm{k}$ & Bias & MSE & Bias & MSE & Bias & MSE \\
\hline 0.2 & 10 & 0.0078 & $0.1225^{*} 10^{-3}$ & 0.0080 & $0.1293^{*} 10^{-3}$ & 0.0081 & $0.1305^{*} 10^{-3}$ \\
& 20 & 0.0078 & $0.1225^{*} 10^{-3}$ & 0.0080 & $0.1293^{*} 10^{-3}$ & 0.0081 & $0.1305^{*} 10^{-3}$ \\
& 40 & 0.0078 & $0.1225^{*} 10^{-3}$ & 0.0080 & $0.1293^{*} 10^{-3}$ & 0.0081 & $0.1305^{*} 10^{-3}$ \\
& 60 & 0.0078 & $0.1225^{*} 10^{-3}$ & 0.0080 & $0.1293^{*} 10^{-3}$ & 0.0081 & $0.1305^{*} 10^{-3}$ \\
& 80 & 0.0078 & $0.1225^{*} 10^{-3}$ & 0.0080 & $0.1293^{*} 10^{-3}$ & 0.0081 & $0.1305^{*} 10^{-3}$ \\
& 100 & 0.0078 & $0.1207^{*} 10^{-3}$ & 0.0080 & $0.1280^{*} 10^{-3}$ & 0.0081 & $0.1299^{*} 10^{-3}$ \\
\hline 0.6 & 10 & 0.0039 & $0.3064^{*} 10^{-4}$ & 0.0040 & $0.3232^{*} 10^{-4}$ & 0.0040 & $0.3262^{*} 10^{-4}$ \\
& 20 & 0.0039 & $0.3064^{*} 10^{-4}$ & 0.0040 & $0.3232^{*} 10^{-4}$ & 0.0040 & $0.0 .3262^{*} 10^{-4}$ \\
& 40 & 0.0039 & $0.3064^{*} 10^{-4}$ & 0.0040 & $0.3232^{*} 10^{-4}$ & 0.0040 & $0.0 .3262^{*} 10^{-4}$ \\
& 60 & 0.0039 & $0.3064^{*} 10^{-4}$ & 0.0040 & $0.3232^{*} 10^{-4}$ & 0.0040 & $0.0 .3262^{*} 10^{-4}$ \\
& 80 & 0.0039 & $0.3064^{*} 10^{-4}$ & 0.0040 & $0.3232^{*} 10^{-4}$ & 0.0040 & $0.0 .3262^{*} 10^{-4}$ \\
& 100 & 0.0039 & $0.3016^{*} 10^{-4}$ & 0.0040 & $0.3200^{*} 10^{-4}$ & 0.0040 & $0.0 .3249^{*} 10^{-4}$ \\
\hline 0.8 & 10 & 0.0020 & $0.7659^{*} 10^{-5}$ & 0.0020 & $0.8081^{*} 10^{-5}$ & 0.0020 & $0.8154^{*} 10^{-5}$ \\
& 20 & 0.0020 & $0.7659^{*} 10^{-5}$ & 0.0020 & $0.8081^{*} 10^{-5}$ & 0.0020 & $0.8154^{*} 10^{-5}$ \\
& 40 & 0.0020 & $0.7659^{*} 10^{-5}$ & 0.0020 & $0.8081^{*} 10^{-5}$ & 0.0020 & $0.8154^{*} 10^{-5}$ \\
& 60 & 0.0020 & $0.7659^{*} 10^{-5}$ & 0.0020 & $0.8081^{*} 10^{-5}$ & 0.0020 & $0.8154^{*} 10^{-5}$ \\
& 80 & 0.0020 & $0.7658^{*} 10^{-5}$ & 0.0020 & $0.8080^{*} 10^{-5}$ & 0.0020 & $0.8154^{*} 10^{-5}$ \\
& 100 & 0.0020 & $0.7541^{*} 10^{-5}$ & 0.0020 & $0.8000^{*} 10^{-5}$ & 0.0020 & $0.8121^{*} 10^{-5}$ \\
\hline
\end{tabular}

Table 2. Variation of the approximate bias and the MSE for $n=100, \alpha=0.2$ and $\rho=$ $0.2,0.6,0.8$
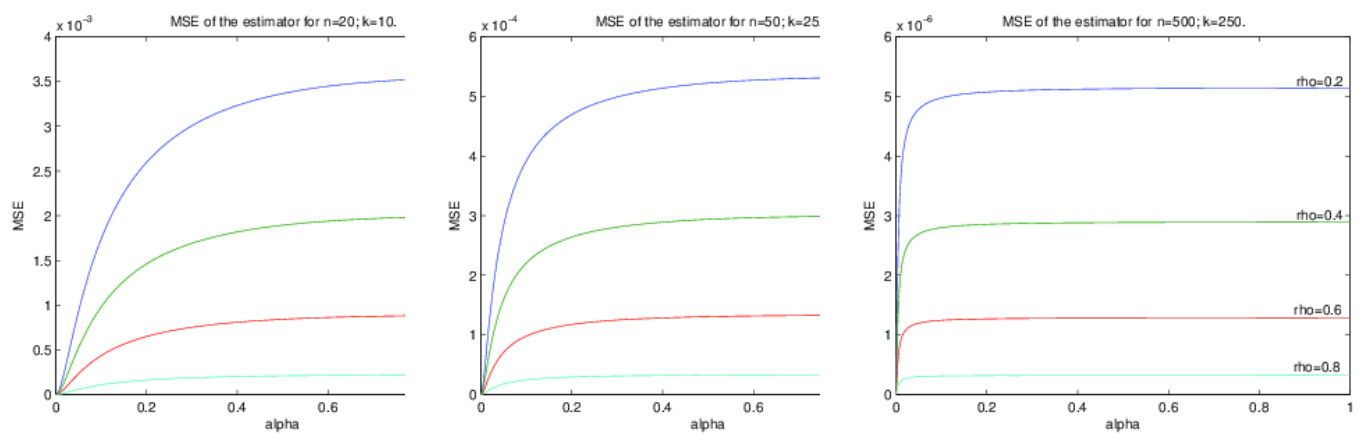

Fig. 2. Variation of the approximate MSE with $\alpha$

This function is called "bias robustness function". In the same way, we define the second measure called the "MSE robustness function" as follows:

$$
\text { rmse }_{\left[\alpha_{1}, \alpha_{2}\right]}(\rho)=\sup _{\alpha_{1} \leq \alpha \leq \alpha_{2}} \widetilde{M S E}(\rho, \alpha)-\inf _{\alpha_{1} \leq \alpha \leq \alpha_{2}} \widetilde{M S E}(\rho, \alpha) .
$$

where $0<\alpha_{1}<\alpha_{2} \leq 1$. The methodology consists in evaluating the maximum oscillation of a given property of a statistic in the supermodel. To calculate the values of these functions, one need the following properties.

Proposition 2. The approximate bias $\widetilde{B}(\rho, \alpha)$ of $\hat{\rho}$ is an increasing function of $\alpha$ when $0<\alpha<1$. 
Proof.

$$
\widetilde{B}(\rho, \alpha)=\frac{\alpha}{\alpha^{2}\left(1+\rho m\left(1-\rho^{n-k-1}\right)\right)+\alpha m\left[(n-3+\rho)-\rho\left(1-\rho^{n-k-1}\right)\right]+m}
$$

The first derivative of $\widetilde{B}(\rho, \alpha)$ according to $\alpha$ is

$$
\widetilde{B^{\prime}}(\rho, \alpha)=\frac{\partial \widetilde{B}(\rho, \alpha)}{\partial \alpha}=\frac{-\alpha^{2}\left(1+\rho m\left(1-\rho^{n-k-1}\right)\right)+m}{\left[\alpha^{2}\left(1+\rho m\left(1-\rho^{n-k-1}\right)\right)+\alpha m\left[(n-3+\rho)-\rho\left(1-\rho^{n-k-1}\right)\right]+m\right]^{2}}
$$

We have

$$
\widetilde{B^{\prime}}(\rho, \alpha)=\frac{\partial \widetilde{B}(\rho, \alpha)}{\partial \alpha}=0 \Longleftrightarrow \alpha^{\prime}=\frac{-1}{\sqrt{1-\rho^{n-k}}}, \quad \alpha "=\frac{1}{\sqrt{1-\rho^{n-k}}}
$$

such that $-\infty<\alpha^{\prime}<0<1<\alpha^{\prime \prime}<+\infty$

$$
\widetilde{B^{\prime}}(\rho, \alpha)=\frac{\partial \widetilde{B}(\rho, \alpha)}{\partial \alpha} \geq 0 \Longleftrightarrow \alpha \in\left[\alpha^{\prime}, \alpha^{\prime \prime}\right] \supset[0,1]
$$

Then, the bias is an increasing function of $\alpha$ in the interval $[0,1] . \diamond$

Proposition 3. The approximate Mean Square Error $\widetilde{M S E}(\rho, \alpha)$ of $\hat{\rho}$ is an increasing function of $\alpha$ when $0<\alpha<1$.

Proof. The first derivative of $\widetilde{M S E}(\rho, \alpha)$ according to $\alpha$ is

$$
\widetilde{M S E^{\prime}}(\rho, \alpha)=\frac{\partial \widetilde{M S E}(\rho, \alpha)}{\partial \alpha}=4 \widetilde{B}(\rho, \alpha) \widetilde{B^{\prime}}(\rho, \alpha)
$$

Since $\widetilde{B}(\rho, \alpha)>0$, when $0<\alpha<1$ (see Proposition 2 ), and because $\widetilde{B}(\rho, 0)=0$, so $\widetilde{B}(\rho, \alpha)>0$, with $0<\alpha<1$. Then

$$
\widetilde{M S E^{\prime}}(\rho, \alpha)=\frac{\partial \widetilde{M S E}(\rho, \alpha)}{\partial \alpha}=4 \widetilde{B}(\rho, \alpha) \widetilde{B^{\prime}}(\rho, \alpha)>0
$$

when $0<\alpha<1$. We conclude that $\widetilde{M} S E(\rho, \alpha)$ is an increasing function of $\alpha$ in the interval $[0,1]$.

\subsection{The robustness bias function.}

We consider the approach of Zieliñski to study the stability of the bias and the MSE.

Following the Proposition 2,

$$
\begin{gathered}
\inf _{0 \leq \alpha \leq 1} \widetilde{B}(\rho, \alpha)=\widetilde{B}(\rho, 0)=0, \\
\sup _{0 \leq \alpha \leq 1} \widetilde{B}(\rho, \alpha)=\widetilde{B}(\rho, 1)=\frac{(1-\rho)}{n-1} .
\end{gathered}
$$

Journal home page: www.jafristat.net 
Then, the robustness bias function

$$
\operatorname{rbias}_{[0,1]}(\rho)=\frac{1-\rho}{n-1}
$$

We can notice that

$$
\begin{aligned}
& \lim _{\rho \rightarrow 1} \operatorname{rbias}_{[0,1]}(\rho)=0, \\
& \lim _{\rho \rightarrow 0} \operatorname{rbias}_{[0,1]}(\rho)=\frac{1}{n-1}
\end{aligned}
$$

and

$$
\lim _{n \rightarrow+\infty} \operatorname{rbias}_{[0,1]}(\rho)=0 .
$$

According to the above remarks, one can say that the maximal oscillation of the bias robustness function is $1 /(n-1)$ when the contaminant $\alpha$ varies from one to zero. This maximal oscillation vanishes in the asymptotic case. Then, we can affirm that the estimator is asymptotically robust with respect to the bias.

\subsection{The robustness MSE function.}

Let be

$$
\widetilde{M S E}(\rho, \alpha)=\frac{2(1-\rho)^{2} \alpha^{2}}{\left[\alpha^{2}\left(1-\rho^{n-k}\right)+\alpha\left(n-3+\rho^{n-k}\right)+1\right]^{2}} .
$$

Following the Proposition 3,

$$
\begin{gathered}
\inf _{0 \leq \alpha \leq 1} \overparen{M S E}(\rho, \alpha)=\widetilde{M S E}(\rho, 0)=0, \\
\sup _{0 \leq \alpha \leq 1} \widetilde{M S E}(\rho, \alpha)=\widetilde{M S E}(\rho, 1)=\frac{2(1-\rho)^{2}}{(n-1)^{2}} .
\end{gathered}
$$

The robustness MSE function is then,

$$
\operatorname{rmse}_{[0,1]}(\rho)=\frac{2(1-\rho)^{2}}{(n-1)^{2}} .
$$

The MSE robustness function presents the same properties than the bias robustness function. So,

$$
\begin{aligned}
& \lim _{\rho \rightarrow 1} \operatorname{rmse}_{[0,1]}(\rho)=0, \\
& \lim _{\rho \rightarrow 0} \operatorname{rmse}_{[0,1]}(\rho)=\frac{2}{(n-1)^{2}}
\end{aligned}
$$

and

$$
\lim _{n \rightarrow+\infty} \operatorname{rmse}_{[0,1]}(\rho)=0 .
$$

Finally, the maximum likelihood estimator is asymptotically robust with respect to the bias and MSE.

Journal home page: www.jafristat.net 
H. Fellag, L. Atil and C. Belkacem, Afrika Statistika, Vol. 9, 2014, pages 647-658. On the stability of estimation of $\mathrm{AR}(1)$ coefficient in the presence of contaminated exponential innovations.

\begin{tabular}{|c|cccc|}
\hline \multirow{2}{*}{$n$} & \multicolumn{4}{|c|}{$\rho$} \\
\cline { 2 - 5 } & 0.2 & 0.4 & 0.6 & 0.8 \\
\hline 20 & 0.410908 & 0.358888 & 0.291902 & 0.195695 \\
\hline 50 & 0.165808 & 0.141448 & 0.112171 & 0.073497 \\
\hline 500 & 0.015981 & 0.013571 & 0.010712 & 0.006985 \\
\hline
\end{tabular}

Table 3. Values of $\alpha_{s}$ for bias function and $\epsilon=0.01$

\begin{tabular}{|c|c|cccc|}
\hline \multirow{3}{*}{$k$} & $n$ & \multicolumn{4}{|c|}{$\rho$} \\
\cline { 3 - 6 } & 20 & 0.2 & 0.4 & 0.6 & 0.8 \\
\hline \multirow{5}{*}{50} & $(0.001792$ & 0.001795 & 0.001749 & 0.001594 \\
& 0.001288 & $(0.000220)$ & $(0.000141)$ & $(0.000062)$ \\
& & 0.001177 & 0.001023 & 0.0007858 \\
& & $(0.000023)$ & $(0.000015)$ & $\left(8.58^{*} 10^{-6}\right)$ & $\left(3.04^{*} 10^{-6}\right)$ \\
& 500 & 0.000173 & 0.000151 & 0.000124 & 0.000088 \\
& & $\left(1.05^{*} 10^{-6}\right)$ & $\left(6.80^{*} 10^{-7}\right)$ & $\left(3.66^{*} 10^{-7}\right)$ & $\left(1.26^{*} 10^{-7}\right)$ \\
\hline
\end{tabular}

Table 4. Values of the bias and MSE robustness function in the interval $\left[\alpha_{s}, 1\right]$

\subsection{Analysis of the results}

If we observe carefully the variation of the bias and MSE, in the previous Figures 1 and 2, including small sample case, one can remark that there is a possible suitable value $\alpha_{s} \in[0,1]$ such that the bias and MSE are stable in the interval $\left[\alpha_{s}, 1\right]$. In the following, we propose to calculate the value $\alpha_{s}$ for the bias. We proceed as follows : for a fixed $\rho, n$ and the position of the contaminant $k$,

- Step 1: Compute $\widetilde{B^{\prime}}(\rho, 1)$ where $\widetilde{B^{\prime}}(\rho, \alpha)$ represents the first derivative of the bias according to $\alpha$.

- Step 2: Choose a maximal variation $\varepsilon$, say $\varepsilon=0.01$, of the bias.

- Step 3: Calculate $\varphi_{\text {bias }}=\left|\widetilde{B^{\prime}}(\rho, \alpha)-\widetilde{B^{\prime}}(\rho, 1)\right|$.

- Step 4: Compute $\alpha_{s}=\min \left\{\alpha \in[0,1]: \varphi_{\text {bias }}<\varepsilon\right\}$.

The analytic expression of $\varphi(\alpha)$ is

$$
\varphi_{\text {bias }}(\alpha)=\left|\frac{-\alpha^{2}(1-\rho)\left(1-\rho^{n-k}\right)+(1-\rho)}{\left[\alpha^{2}\left(1-\rho^{n-k}\right)+\alpha\left(n-3+\rho^{n-k}\right)+1\right]^{2}}-\frac{(1-\rho) \rho^{n-k}}{(n-1)^{2}}\right|
$$

The Table 3 presents some values of $\alpha_{s}$ obtained, using numerical approximations, for $\rho=$ $0.2,0.4,0.6,0.8, n=20,50,500$. Without loss of generality, the position $k$ of the contaminant is chosen to be occurring at the position $[n / 2]$.

After these computations of $\alpha_{s}$, we obtain the values of the bias and MSE robustness functions given in Table 4, the values of the MSE robustness function are in brackets.

It is easy to see that the robustness functions are very close to zero in the interval $\left[\alpha_{s}, 1\right]$, even for small sample cases. This can be considered as a nice property of the maximum likelihood estimator of $\rho$ under this kind of contamination. 
H. Fellag, L. Atil and C. Belkacem, Afrika Statistika, Vol. 9, 2014, pages 647-658. On the stability of estimation of $\mathrm{AR}(1)$ coefficient in the presence of contaminated exponential innovations.

If we consider the robustness functions of the bias and MSE with respect to the parameter $\rho$, the formulas (16) and (17) allow us to say that:

(i) The bias robustness function is equal to twice the square of the MSE robustness function, for every $\rho$ and every $n$. i.e

$$
\left.\operatorname{rmse}_{[0,1]}(\rho)=2 * \operatorname{rbias}_{[0,1]}(\rho)\right)^{2}
$$

(ii) The robustness function decreases when $\rho$ grows from zero to one as given in the following figure :
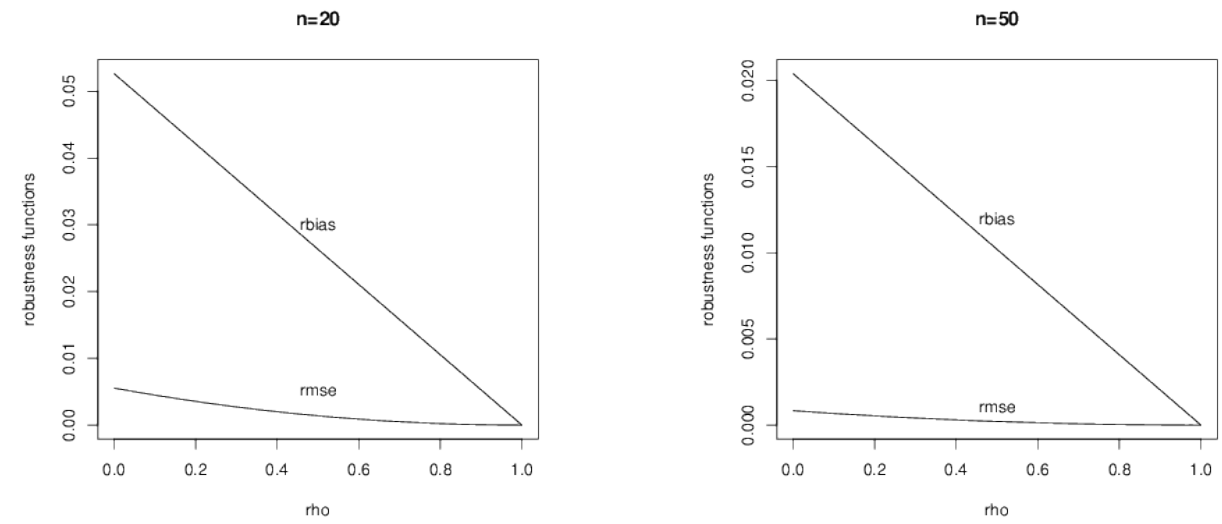

Fig. 3. Variation of the robustness functions of the bias with $\rho$

Then, for every sample size $n$, the maximal oscillation of the bias and the MSE for all the values of $\rho$ are

$$
\max _{\rho \in[0,1]} \operatorname{rbias}_{[0,1]}(\rho)=1 /(n-1) \quad \text { and } \quad \max _{\rho \in[0,1]} \operatorname{rmse}_{[0,1]}(\rho)=1 /(n-1)^{2}
$$

\section{Concluding remarks}

In this paper, we studied the behavior of the MLE of a first-order autoregressive parameter $\rho$ under a kind contamination proposed by Kale and Sinha (1969) in the identically independent distribution case. We proved that the methodology of Andẽl (1988) is valid under this contaminated model. Finally, we showed that the MLE is asymtotically robust with respect to the bias and MSE and is stable if the contaminant $\alpha$ varies between a suitable value $\alpha_{s}$ computed numerically and one, even in small sample case. To improve this work, one can consider other types of contamination.

Acknowledgments. Thans for the referees for their helpful comments.

Journal home page: www.jafristat.net 
H. Fellag, L. Atil and C. Belkacem, Afrika Statistika, Vol. 9, 2014, pages 647-658. On the stability of estimation of $\mathrm{AR}(1)$ coefficient in the presence of contaminated exponential innovations. $\quad 658$

\section{References}

An, H.-Z. and Huang, F. 1993. Estimation for regressive and autoregressive models with non-negative residual errors. Journal of Time Series Analysis. 14, 179-191.

Andẽl, J. 1988. On AR(1) processes with exponential white noise. Comm. statist., A, Theory and methods. 17(5), 1481-1495.

Andẽl, J. 1989. Non-negative autoregressive processes. Journal of Time Series Analysis. 10, $1-12$.

Andẽl, J and Zvãra, K., 1988. Table for AR(1) processes with exponential white noise. Kibernitika. 24(5), 372-377.

Davis, R.A. and McCormick, W.P., 1989. Estimation for the first-order autoregressive processes with positive or bounded innovations. Stochastic Process and Applications. 31, 237-250.

Bell, C.B and Smith, E.P., 1986. Inference for non negative autoregressive schemes. Communication in statistics. Theory and methods 15, 2267-2293.

Fellag, H., 2001. Testing on the first order autoregressive model with contaminated exponential white noise. Finite sample case, Discussiones Mathematicae. Probability and Statistics. 21(1), 11-20.

Fellag, H. and Ibazizen, M., 2001. Estimation of the first-order autoregressive model with contaminated exponential innovations. Journal of Mathematical Sciences. 106(1), 26522656.

Fellag, H. and Ibazizen, M., 2003. Bayesian Analysis of AR(1) model with exponential white noise. Statistics. 37(5), 365-372.

Gaver, D.P. and Lewis, P.A.W., 1980. First-order autoregressive Gamma sequences and point process. Adv. Appl. Prob. 12,727-745.

Kale, B.K. and Sinha, S.K., 1969. Estimation of expected life in the presence of an outlier observation. Techometric. 13(4), 755-759.

Nielsen, B. and Shephard, N., 2003. Likelihood analysis of a first order autoregressive model with exponential innovations, Journal of Time Series Analysis. 24, 337-344.

Turkman, M.A.A., 1990. Bayesian analysis of an autoregressive process with exponential white noise. Statistics. 21(4), 601-608.

Zieliñski, R. 1977. Robustness: A quantitative approach. Bulletin de l'Académie Polonaise des sciences, Série sciences Math., Astronomie et physique XXV. 12,1281-1286. 